\title{
Prediction of Belimumab Pharmacokinetics in Chinese Pediatric Patients with Systemic Lupus Erythematosus
}

\author{
Xuan Zhou' ${ }^{1}$ Tsung-I Lee ${ }^{1} \cdot$ Min Zhu $^{1,2} \cdot$ Peiming Ma ${ }^{1}$ (1)
}

Accepted: 7 September 2021 / Published online: 9 October 2021

(c) The Author(s) 2021

\begin{abstract}
Background and Objective Intravenous (IV) belimumab is the first treatment approved for children $\geq 5$ years of age with active autoantibody-positive systemic lupus erythematosus (SLE) in the USA, Europe, and Japan. Pharmacokinetic data for belimumab were collected from several clinical trials in Chinese and non-Chinese adults and non-Chinese pediatric patients with SLE. This study aimed to predict the belimumab dose-exposure relationship to Chinese pediatric patients with SLE, as part of the belimumab registration process for this population in China, using a population PK modeling approach.

Methods An initial linear two-compartment population pharmacokinetic model was built using data from adults only, and considering and adjusting for the covariates age, body weight, body mass index, fat-free mass, race, baseline albumin and immunoglobulin G levels. The model was used to study possible ethnic differences between Chinese and non-Chinese adults and to predict pediatric pharmacokinetic data in a study of non-Chinese pediatric patients (PLUTO study; NCT01649765). The predicted data were compared with the observed data from PLUTO. The model was then updated with pediatric data from PLUTO to predict steady-state belimumab exposure in Chinese pediatric patients with SLE receiving belimumab 10 $\mathrm{mg} / \mathrm{kg}$ IV every 4 weeks.

Results The dataset comprised 9650 sampled concentration values from 1783 patients. The pharmacokinetics of belimumab were adequately described by the final model using all adult and pediatric data with the estimated typical clearance of $238 \mathrm{ml} /$ day in adult and pediatric patients and steady-state volume of distribution of $4915 \mathrm{ml}$ in adults. Between-patient variability was modest (coefficients of variation: $26.1 \%$ for clearance; $8.9 \%$ and $28.5 \%$, respectively, for volumes of distribution of the central and peripheral compartments). Six covariates were identified that influenced pharmacokinetics: age, fat-free mass, an indicator of North East Asian race, baseline albumin, immunoglobulin G, and an early study indicator (two early phase I and phase II belimumab studies: LBSL01 and LBSL02). The analysis showed no apparent difference in steady-state exposure between Chinese and non-Chinese populations and between pediatric and adult populations receiving belimumab $10 \mathrm{mg} / \mathrm{kg}$ IV.

Conclusions In Chinese pediatric patients with SLE, belimumab $10 \mathrm{mg} / \mathrm{kg}$ IV every 4 weeks is expected to have exposure similar to that in Chinese adults and non-Chinese pediatric patients with SLE, supporting the use of this regimen in Chinese pediatric patients with SLE.

Clinical Trial Registration Numbers NCT01649765, NCT00657007, NCT00071487, NCT01345253, NCT01516450, NCT00410384, NCT00424476, NCT02880852, NCT01583530.
\end{abstract}

Peiming Ma

peimingma@gmail.com

1 Clinical Pharmacology Modeling and Simulation, GSK, GlaxoSmithKline, 999 Huanke Road, Pudong, Shanghai 201203, China

2 Department of Pharmacy, Shanghai Chest Hospital, Shanghai Jiao Tong University, Shanghai, China 


\section{Key Points}

Intravenous (IV) belimumab $10 \mathrm{mg} / \mathrm{kg}$ every 4 weeks is approved for children aged $\geq 5$ years with active autoantibody-positive systemic lupus erythematosus (SLE) in the USA, Europe, and Japan based on the results from a phase II efficacy study (PLUTO study) in pediatric patients and other studies in adults. However, no data were available from Chinese pediatric patients, and belimumab was not approved for the treatment of pediatric patients with SLE in China at the time of this study.

Previous population pharmacokinetic analyses using data from PLUTO or a phase III study in North East Asian patients (BEL113750) suggested that, under the 10-mg/ $\mathrm{kg}$ IV dosing regimen, belimumab exposures in Chinese adult patients $(N=42)$ were around 50\% lower than those in pediatric patients of North East Asian heritage $(N=3)$ from the PLUTO study. This led to the question of how, for the same dosing regimen, exposures would compare between Chinese pediatric and adult patients, and between Chinese and non-Chinese pediatric patients.

This study developed a population pharmacokinetics model to predict drug exposure in Chinese pediatric patients using data from the previous belimumab studies to support an indication extension for belimumab in Chinese pediatric patients with SLE. The results of this study supported the use of belimumab $10 \mathrm{mg} / \mathrm{kg}$ IV every 4 weeks in Chinese pediatric patients with SLE.

\section{Introduction}

Belimumab is a human immunoglobulin $\mathrm{G}$ (IgG) $1 \lambda$ monoclonal antibody targeting soluble B-cell activating factor (also known as B-lymphocyte stimulator [BLyS]), a potent B-cell survival factor that correlates with disease activity in patients with systemic lupus erythematosus (SLE) [1-3]. The clinical development program in patients with SLE showed that belimumab was well-tolerated and was associated with reduced SLE disease activity, fewer severe flares, and lower prednisone use than was placebo [4-7]. Intravenous (IV) belimumab is currently approved for the treatment of autoantibody-positive SLE in adult patients in the USA, all European Economic Area countries, Japan, China, Russia, Argentina, and Brazil [8-11] with a dosing regimen of a $10-\mathrm{mg} / \mathrm{kg}$ infusion at 2-week intervals for the first three doses and at 4-week intervals thereafter. Based on the positive benefit-risk ratio in children aged 5-17 years [8, 11-14], belimumab $10 \mathrm{mg} / \mathrm{kg}$ IV is also approved for the treatment of pediatric SLE in the USA, Europe, and Japan.

The efficacy and safety of belimumab in adult patients with SLE from North East Asia was established in a phase III study (GlaxoSmithKline [GSK] study BEL113750; NCT01345253) that included 517 adult Chinese patients with SLE [7]. In addition, a phase I study (GSK study 200909; NCT02880852) further evaluated the pharmacokinetics, pharmacodynamics, and safety of belimumab in adult Chinese patients [15]. These data supported the use of belimumab in the adult SLE population in China; however, belimumab was not yet approved for the treatment of pediatric patients with SLE in China at the time of this study.

Drug development in pediatric patients has a variety of obstacles, including the scarcity of this study population and the difficulty and complexity of conducting studies. The ethical considerations also differ. Efforts should generally be made to use adult efficacy data based on extrapolation exercises that include factors such as body size, age, and other maturation-related variables [16]. Similar exposures were observed between non-Chinese adult and pediatric patients. However, data from the BEL113750 (NCT01345253) and PLUTO (GSK Study BEL114055; NCT01649765) studies suggested that belimumab exposures in adult Chinese patients were lower than those in pediatric patients of Asian heritage based on the $10-\mathrm{mg} / \mathrm{kg}$ IV dosing regimen [13, 15]. Thus, uncertainty remains surrounding the expected exposures of belimumab $10 \mathrm{mg} / \mathrm{kg}$ IV in Chinese pediatric patients with SLE.

A proposal to extend the indication for belimumab to include Chinese pediatric patients aged 5-17 years can be based on results from data prediction to the pediatric population. This study aimed to support this approach by developing a population pharmacokinetic model using data from key belimumab studies to predict drug exposure in Chinese pediatric patients with SLE by estimating exposure and pharmacokinetic parameters from Chinese/non-Chinese adult and non-Chinese pediatric patients with SLE.

\section{Methods}

\subsection{Objectives}

The objectives of this study were to develop a population pharmacokinetic model that could identify the potential effect of selected covariates (e.g., age, race, body size variables, baseline patient characteristics) on pharmacokinetic parameters and to compare the model-predicted exposure of IV and subcutaneous (SC) belimumab in Chinese and non-Chinese healthy participants and patients with SLE. This population pharmacokinetic analysis comprises the 


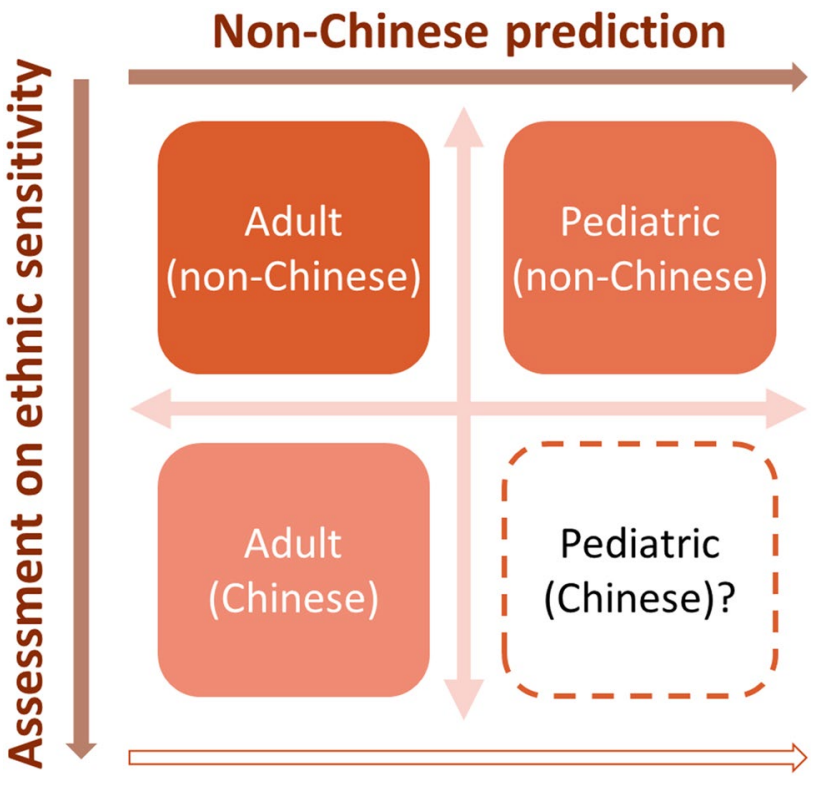

Fig. 1 Pediatric prediction scheme from Chinese adult and non-Chinese adult and pediatric patients

dose-exposure part of the prediction to the Chinese pediatric population based on pharmacokinetic data from Chinese and non-Chinese adults and pediatric patients with SLE (Fig. 1).

\subsection{Study Design and Endpoints}

Pharmacokinetic data were collected from healthy participants and patients with SLE from nine prospective clinical belimumab studies (BEL114055 [PLUTO; NCT01649765], LBSL01 [NCT00657007], LBSL02 [NCT00071487], BEL113750 [BLISSNEA; NCT01345253], BEL116119 [NCT01516450], BEL110751 [BLISS-76; NCT00410384], BEL110752 [BLISS-52; NCT00424476], 200909 [NCT02880852], BEL114448 [NCT01583530]) in Chinese and non-Chinese adults with SLE, non-Chinese pediatric patients with SLE, and non-Chinese healthy volunteers (Table $\mathrm{S} 1$ in the electronic supplementary material [ESM]) for a population pharmacokinetic analysis.

\subsubsection{Assay Methods}

Three assay methods were used to determine serum belimumab concentrations. Enzyme-linked immunosorbent assay was used in studies LBSL01 and LBSL02, with a lower limit of quantification (LLOQ) of $138.5 \mathrm{ng} / \mathrm{ml}$ of belimumab in human serum. An electrochemiluminescence-based assay with an LLOQ of $100 \mathrm{ng} / \mathrm{ml}$ was used in BLISS-52 and BLISS-76. The PLUTO study and studies BEL113750, BEL116119, 200909 and BEL114448 also used an electrochemiluminescence-based assay; in these studies, serum samples were analyzed in two laboratories and the method was cross-validated prior to analysis.

\subsection{Modeling and Simulation}

\subsubsection{Software and Estimation Methods}

A nonlinear mixed-effects model was developed using the software NONMEM ${ }^{\circledR}$, version 7.3 (ICON Development Solutions). The parameter estimation used the ADVAN3 TRANS4 subroutine and the first-order conditional estimation method with interaction term based on the previously characterized IV population pharmacokinetic model [17]. $\mathrm{R}$ version 3.6.0 was used for generating model diagnostic plots and revising the datasets needed for population pharmacokinetic analyses. Quality control steps were undertaken throughout the work process to assemble the datasets and check for accuracy.

\subsubsection{Handling of Missing Data and Outliers}

All values below the LLOQ were excluded from the analysis, and no other data were removed. No concentration or covariate data were imputed for missing values before the analysis. Graphical exploration of data was undertaken to identify outliers. The goodness-of-fit plots were used to decide on the inclusion or exclusion of the outliers in the final data analysis.

\subsubsection{Population Pharmacokinetic Model Development}

Based on previous pharmacokinetic analyses of belimumab in patients with SLE at dose regimens $>1 \mathrm{mg} / \mathrm{kg}$ every 4 weeks (Q4W), belimumab does not display nonlinearity due to target-mediated drug disposition, a phenomenon typical of monoclonal antibodies $[17,18]$. The pharmacological effects of belimumab are mediated via its binding to and neutralization of soluble BLyS, which inhibits B-cell survival and differentiation without directly causing B-cell death. Average belimumab concentrations at steady state $\left(C_{\text {avg_ss }}\right)$ are approximately $100 \mu \mathrm{g} / \mathrm{ml}$ for the $10 \mathrm{mg} / \mathrm{kg} \mathrm{IV}$ and $200 \mathrm{mg} \mathrm{SC}$ dosing regimens. There is a significant molar excess of belimumab at these exposures, at approximately 25,000 times higher concentrations than with the BLyS trimer; as such, BLyS inhibition is expected to be saturated [18]. A linear two-compartment structural model was initially used that included interindividual variability in clearance $(\mathrm{CL})$, intercompartmental clearance $(Q)$, 
and volumes of distribution of the central and peripheral compartments (V1 and V2, respectively). A combination of additive and multiplicative terms was considered for the residual error of the model. The model selection was based on evaluation of minimum objective function values (OFV), goodness-of-fit, and precision of parameter estimates.

The initial model was built using pharmacokinetic data from adult patients only to study pharmacokinetic differences between Chinese and non-Chinese patients with SLE. Potential covariates that might affect pharmacokinetics were investigated. For monoclonal antibodies, multiple mechanisms exist by which the disease state or target binding can affect pharmacokinetics. For example, the neonatal $\mathrm{Fc}$ receptor $(\mathrm{FcRn})$ recycles and thus protects IgG from lysosomal degradation. Endogenous IgG competes with the therapeutic antibodies for FcRn binding. The level of albumin may be an indicator of FcRn expression. A high albumin level implies a high degree of protection of IgG from degradation and reduced CL of a monoclonal antibody [19]. Therefore, the effects of baseline $\mathrm{IgG}$ and baseline albumin on pharmacokinetics were evaluated. The effects of the following demographic and baseline variables were also investigated for their influence on the main pharmacokinetic parameters: race variables (Chinese [ $n=135]$ vs. non-Chinese [ $n=1648]$; North East Asians [i.e., worldwide Chinese, Japanese, and Koreans $\{n=235\}]$ vs. non-North East Asians [ $n=1548]$ ); body size (body weight, body mass index, fat-free mass [FFM]); age and sex; and baseline albumin and IgG levels. FFM was calculated using the formulae from Janmahasatian et al. [20]. Covariate selection was guided by biological plausibility with any of the pharmacokinetic parameters and by the decrease in the minimum OFV, approximated as a $\chi^{2}$ distribution, using a combined forward selection and backward elimination approach. In building the model with the forward selection, the decrease in the minimum OFV was set to be greater than 3.84 units for the addition of a single covariate parameter $(p<0.05)$. Covariates were added manually to the base model one by one, starting with the most significant, until the full model for which none could be further added according to the criteria. From the full model, the significance of each covariate was tested individually by removing one at a time until all non-significant covariates were excluded. A covariate was retained if, upon removal, the minimum OFV increased by more than 10.83 units $(p<0.001)$. The backward elimination continued until all covariates in the model passed the criteria described. The model confirmed by backward elimination was the final model.

The established model was used to predict pediatric pharmacokinetic data from the PLUTO study using their baseline covariates and collected dosing and sampling times. The predicted data were then compared with the observed concentrations, and if they were consistent, the model was updated with pediatric data from PLUTO. Otherwise, possible causes of inconsistency were investigated. The final model was used to predict pharmacokinetic and exposure parameters in Chinese pediatric patients with SLE under the dosing regimen of $10 \mathrm{mg} / \mathrm{kg}$ Q $4 \mathrm{~W}$. The predicted exposures were compared with those of non-Chinese pediatric and Chinese adult patients with SLE.

\subsubsection{The Criterion for Pharmacokinetic and Exposure Comparison}

To justify the appropriateness of the dosing regimen $(10 \mathrm{mg} / \mathrm{kg} \mathrm{Q} 4 \mathrm{~W})$, a similarity criterion was used for comparisons of pharmacokinetic parameters and exposure distributions between Chinese and non-Chinese adult populations, non-Chinese adult and pediatric populations, and Chinese adult and pediatric populations. The exposure distribution for the Chinese pediatric patients was predicted, while for other populations it was based on empirical Bayes estimates. The individual steady-state exposure values ( $C_{\text {avg_ss }}$; predicted from the final model under the dosing regimen of $10 \mathrm{mg} / \mathrm{kg} \mathrm{Q} 4 \mathrm{~W}$ ), either calculated post hoc or simulated, were first generated using the best population pharmacokinetic model. The two distributions were considered similar if the $80 \%-125 \%$ calculated interval of the first and third quartiles of one distribution included those of another. The scalers 80 and $125 \%$ were selected based on common acceptable concepts of pharmacokinetic and exposure parameter variability (e.g., in the bioequivalence acceptance criterion) [21]. More standard statistical comparison of the two distributions (e.g., Kolmogorov-Smirnov test) was not considered necessary as the purpose was to assess or match the two distributions under the context of belimumab therapeutic windows.

\section{Results}

\subsection{Population Pharmacokinetics}

The dataset used for the population pharmacokinetic analysis consisted of 9650 concentration values from samples collected from 1783 patients from nine studies (Table 1). The analysis population consisted of 1730 adult and 53 pediatric patients (age range $6-80$ years). Overall, 1979 of 11,629 (17.0\%) pharmacokinetic samples were below the LLOQ and therefore excluded from the data analysis. 
Table 1 Participant demographics and baseline variables, $N=1783$

\begin{tabular}{lll}
\hline Demographics and variables & Pediatric participants $(n=53)$ & Adult participants $(n=1730)$ \\
\hline Median (min, max) Age, years ${ }^{\mathrm{a}}$ & $14(6 ; 18)$ & $38(18 ; 80)$ \\
Median (min, max) Weight, kg & $52.5(17 ; 85.5)$ & $65.7(35.8 ; 165.4)$ \\
Median (min, max) BMI, kg/m² & $21.37(15.42 ; 34.03)$ & $24.82(14.32 ; 56.23)$ \\
Median (min, max) FFM, kg & $34.45(12.56 ; 57.16)$ & $41.08(24.32 ; 87.93)$ \\
Race, n (\%) & & \\
North East Asian & $3(5.66)$ & $232(13.41)$ \\
Other & $50(94.34)$ & $1498(86.59)$ \\
Sex, n (\%) & & $115(6.65)$ \\
Male & $4(7.55)$ & $1615(93.35)$ \\
Female & $49(92.45)$ & $14.7(3.33 ; 61.9)$ \\
Median (min, max) Serum IgG, g/l & $14.5(4.08 ; 31.2)$ & $40(19 ; 55)$ \\
Median (min, max) Albumin, g/l & $43(29 ; 52)$ & \\
\hline
\end{tabular}

$B M I$ body mass index, FFM fat-free mass, $I g G$ immunoglobulin $\mathrm{G}$

${ }^{a}$ One 18-year-old patient was included in PLUTO but counted in the adult category

${ }^{\mathrm{b}}$ Population other than North East Asian

\subsection{Model Development}

Comparison of model predictions in pediatric patients from PLUTO based only on adult data with observed concentrations in the study revealed no great discrepancy other than some underprediction (Fig. 2; left panel). When pharmacokinetic data from PLUTO were also included, only age was identified as a significant covariate on V1. The final model with all data improved the pharmacokinetic prediction in pediatric patients with fewer underpredictions (Fig. 2; right panel) and described the pharmacokinetics of belimumab in adult and pediatric patients adequately. The parameter estimates for a typical patient in the respective datasets from the final model using all data were overall consistent with those using only the adult data, and the variability estimates for CL and V1 were expectedly smaller because of the inclusion of the covariates.

\subsection{Pharmacokinetics and Covariates}

Estimated pharmacokinetic parameters are listed in Table 2. Little bias was seen in the goodness-of-fit plots, indicating that the model described the data well (Fig. 3).

The estimated typical clearance was $238 \mathrm{ml} /$ day in both adult and pediatric populations, and the steady-state volume of distribution (Vss) was $4915 \mathrm{ml}$ in adult patients. Between-patient variability was modest, with coefficients of variation of $26.1 \%$ for clearance, $8.9 \%$ for $\mathrm{V} 1$, and $28.5 \%$ for V2. Important baseline variables such as FFM, albumin, and $\operatorname{IgG}$ that were included in previous population analyses
$[17,18,22]$ as covariates to the pharmacokinetic parameters improved the model fitting to the data, as expected. Examination of goodness-of-fit plots showed that the model overpredicted concentrations from the two early belimumab studies (LBSL01 and LBSL02) (Fig. S1 in the ESM). Possible causes for the apparently lower concentrations could be differences in operation or bioanalytical methods, and assay methods. However, as suggested by Struemper et al. [17], none of these potential factors could be quantified because of their mutual confounding effect. The population pharmacokinetic models of belimumab were, therefore, set up such that the default population pharmacokinetic parameters reflected the exposure in the pivotal phase III studies BEL110751 and BEL110752 as well as the North East Asia phase III study BEL113750, which used product manufactured and formulated in the same manner as the marketed product. The "fudge factor" was thus included so that the data from the two studies could still be used. An indicator variable that differentiated the earlier studies from the later studies was found to be significant when implemented on both CL and V1.

No apparent difference in CL and a moderate difference in V1 were seen between North East Asians and other populations. The other race variables (e.g., Chinese vs. nonChinese) did not show a better fit (similar influence on V1), as judged with the Akaike information criterion [23]. This implies that there was no apparent difference in pharmacokinetics between Chinese and other North East Asians after accounting for body size differences.

The performance of the model was evaluated using visual predictive checks in addition to other model diagnostic tools 

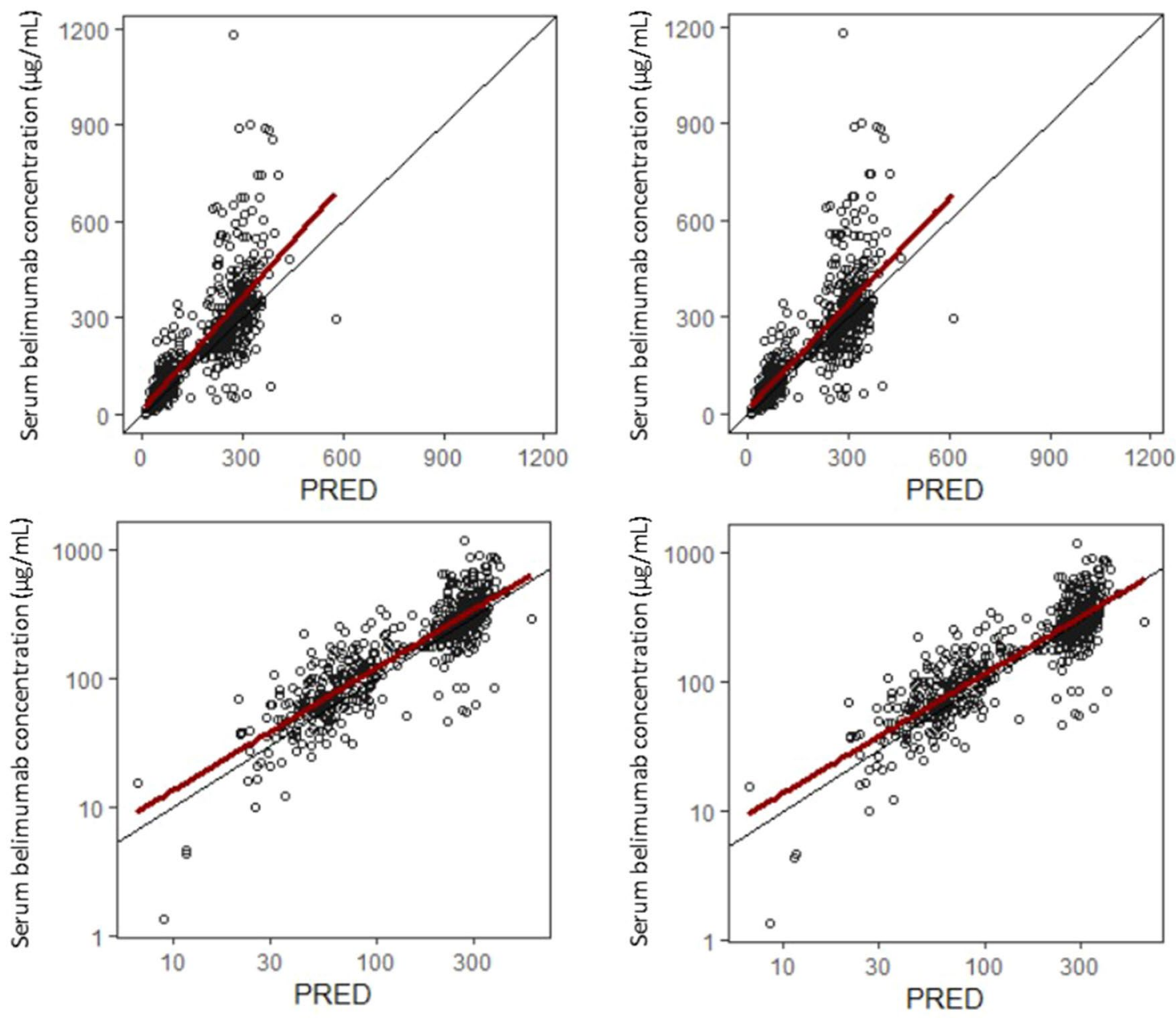

Fig. 2 Model predictions vs. observations in pediatric patients from PLUTO. Left: run607 (adult data only); right: run613 (final model); top: normal scale; bottom: log scale. PRED predicted

such as goodness-of-fit plots. Estimated fixed and random effects were used to simulate 2000 replicates of the final dataset in NONMEM, and Fig. 4 displays the visual predictive check plots with $90 \%$ prediction intervals for belimumab concentrations after the first dose of $10 \mathrm{mg} / \mathrm{kg}$. The observed data were overlaid on the simulations. The model appeared to predict the majority of the data well, but it slightly underpredicted the pediatric data.

\subsection{Distribution of Steady-State Average Concentration ( $\mathrm{C}_{\text {avg_ss }}$ ) for Pediatric and Adult Patients}

The median level of individual $C_{\text {avg_ss }}$ in Chinese adult patients $(N=135)$ was $82.4 \mathrm{mg} / \mathrm{l}$, which was $39.6 \%$ lower than those in pediatric patients of North East Asian heritage $(N=3)$ from the PLUTO study $(136.5 \mathrm{mg} / \mathrm{l})$. The exposure difference between Chinese and non-Chinese patients and between adult and pediatric patients, as well as the influence of covariates on belimumab exposure, was further assessed through simulations. The simulated $C_{\text {avg_ss }}$ under the dosing regimen of $10 \mathrm{mg} / \mathrm{kg}$ Q4W was compared among different racial groups. Consistent with the lack of an effect of race on CL in the model, the results showed no apparent difference in exposure between North East Asian and nonNorth East Asian populations (Fig. 5a) or between Chinese and non-Chinese populations (Fig. 5b). For pediatric and adult patients, following IV administration of belimumab $10 \mathrm{mg} / \mathrm{kg}$ (Fig. $5 \mathrm{c}, \mathrm{d}$ ), the first and third quartiles of $C_{\text {avg_ss }}$ in the overall pediatric population $(78.2$ and $105.8 \mathrm{mg} / \mathrm{l}$, respectively) were within the $80-125 \%$ calculated interval limits for the overall and North East Asian adult populations, indicating overall similarity of exposure distribution (Table 3). 
Table 2 Population pharmacokinetic parameters (final model)

\begin{tabular}{|c|c|c|c|c|c|c|c|c|}
\hline \multirow{2}{*}{$\frac{\text { Description }}{\text { CL (ml/day) }}$} & \multicolumn{2}{|l|}{ Typical value } & \multirow{2}{*}{$\frac{\operatorname{RSE}^{\mathrm{a}}(\%)}{1.2}$} & \multicolumn{2}{|c|}{ Variance $(\eta)$} & \multirow{2}{*}{$\frac{\operatorname{RSE}(\%)}{5.2}$} & \multirow{2}{*}{$\begin{array}{l}\text { Shrinkage (\%) } \\
7\end{array}$} & \multirow{2}{*}{$\frac{\mathrm{CV}^{\mathrm{b}}(\%)}{26.1}$} \\
\hline & $\theta_{1}$ & 238 & & $\eta_{1}$ & $0.0682^{c}$ & & & \\
\hline FFM & $\times(\mathrm{FFM} / 40.69)^{\theta 7}$ & 0.673 & 5.9 & & & & & \\
\hline BALB & $\times(\mathrm{BALB} / 40)^{\theta 9}$ & -1.12 & 6.2 & & & & & \\
\hline BIGG & $\times(\mathrm{BIGG} / 14.8)^{\theta 10}$ & 0.293 & 7.4 & & & & & \\
\hline STDY $=$ LBSL01 or 02 & $\times \theta_{11}^{\mathrm{INDR}}$ & 1.63 & 1.6 & & & & & \\
\hline $\mathrm{V} 1(\mathrm{ml})$ & $\theta_{2}$ & 2597 & 1.5 & $\eta_{2}$ & $0.0079^{c}$ & 36.6 & 35.8 & 8.9 \\
\hline FFM & $\times(\mathrm{FFM} / 40.69)^{\theta 8}$ & 0.891 & 4.2 & & & & & \\
\hline STDY $=$ LBSL01 or 02 & $\times \theta_{12}^{\text {INDR }}$ & 1.26 & 1.4 & & & & & \\
\hline Race $\left(\mathrm{RAC} 4^{\mathrm{d}}\right)$ & $\times \theta_{12}^{\mathrm{RAC} 4}$ & 1.07 & 1.7 & & & & & \\
\hline AGE & $\times \mathrm{AGE} /\left(\mathrm{AGE}+\theta_{14}\right)$ & 1.58 & 33.9 & & & & & \\
\hline$Q$ (ml/day) & $\theta_{3}$ & 591 & 6.5 & $\eta_{3}$ & 0 (fixed) & - & - & - \\
\hline V2 (ml) & $\theta_{4}$ & 2318 & 3.4 & $\eta_{4}$ & 0.0781 & 23.9 & 52 & 28.5 \\
\hline$\varepsilon_{1}(\%)^{\mathrm{e}}$ & $\theta_{5}$ & 0.247 & 1.8 & $\eta_{5}$ & 0.186 & 12.4 & 7.8 & 45.2 \\
\hline$\varepsilon_{2}(\mathrm{mg} / \mathrm{l})^{\mathrm{e}}$ & $\theta_{6}$ & 0.221 & 7.7 & & & & & \\
\hline
\end{tabular}

$A G E$ patient age, $B A L B$ baseline albumin, $B I G G$ baseline immunoglobulin $\mathrm{G}, C L$ clearance, $C V$ coefficient of variance, $F F M$ fat-free mass, $I N D R$ study indicator (INDR $=1$ for study LBSL01 and LBSL02 INDR $=0$ for the rest of studies), $\eta$ interindividual variance, $Q$ intercompartmental clearance, $R A C$ race, $R S E$ relative standard error, $S E$ standard error, $S T D Y$ early study indicator, $\theta$ corresponding structural model parameter, $V 1$ central volume distribution, $V 2$ peripheral volume distribution

${ }^{\mathrm{a}} \mathrm{RSE}(\%)$ is calculated as SE/ltypical valuel $\times 100$

${ }^{\mathrm{b}} \mathrm{CV}(\%)$ is calculated as $\operatorname{sqrt}(\exp (\operatorname{var}(\eta))-1) \times 100$

${ }^{\mathrm{c}} \mathrm{Cov}(\mathrm{CL}, \mathrm{V} 2)=0.0125$ and its $\mathrm{RSE}=22.5 \%$

${ }^{\mathrm{d}} \mathrm{RAC} 4=1$ for North East Asian population and RAC4 $=0$ for other populations

${ }^{\mathrm{e}} \varepsilon_{1}$ is proportional error, $\varepsilon_{2}$ is additive error

\section{Discussion}

This population pharmacokinetic analysis used data from nine clinical IV belimumab studies to predict the pharmacokinetics of belimumab in Chinese pediatric patients with SLE. The study found that several baseline variables (FFM, IgG, and albumin) impacted the pharmacokinetics of belimumab. Early population analyses showed similar pharmacokinetics and exposure between healthy adults and adult patients with SLE under the same dosing regimens $[17,18,22]$; differences between these two groups were not studied in this analysis. There was no great disparity in exposure between Chinese and non-Chinese patients or between pediatric and adult patients with SLE. With the $10-\mathrm{mg} / \mathrm{kg}$ intravenous Q4W dosing regimen, the predicted $C_{\text {avg_ss }}$ in Chinese pediatric patients with SLE showed distributions similar to those in adult patients with SLE (Table 3, Fig. 5). Therefore, this population pharmacokinetic analysis demonstrated that dose-exposure relationships with the $10 \mathrm{mg} / \mathrm{kg}$ IV Q4W dosing regimen in Chinese pediatric patients with SLE should be similar to those in
non-Chinese pediatric patients with SLE and Chinese adult patients with SLE.

Previous population pharmacokinetic analyses suggested that belimumab exposures in Chinese adult patients from study BEL113750 were around 50\% lower than those in pediatric patients of North East Asian heritage in study BEL114055 (data not shown). The current model overpredicted pharmacokinetic exposures of patients in study BEL113750 (Fig. S1 in the ESM). A possible reason for this is the sparse nature of the pharmacokinetic sampling in the small group of patients in study BEL113750. However, the model did fit well to the extensively sampled pharmacokinetic data from the phase I 200909 study in Chinese adult patients with SLE [15], which suggests that exposures from study BEL113750 might not be representative of exposures in Chinese patients.

The pharmacokinetic exposures of Chinese patients were slightly lower than those of other North East Asian populations. Although estimating the categorized CL and V1 in Chinese, non-Chinese, North East Asian, and other 

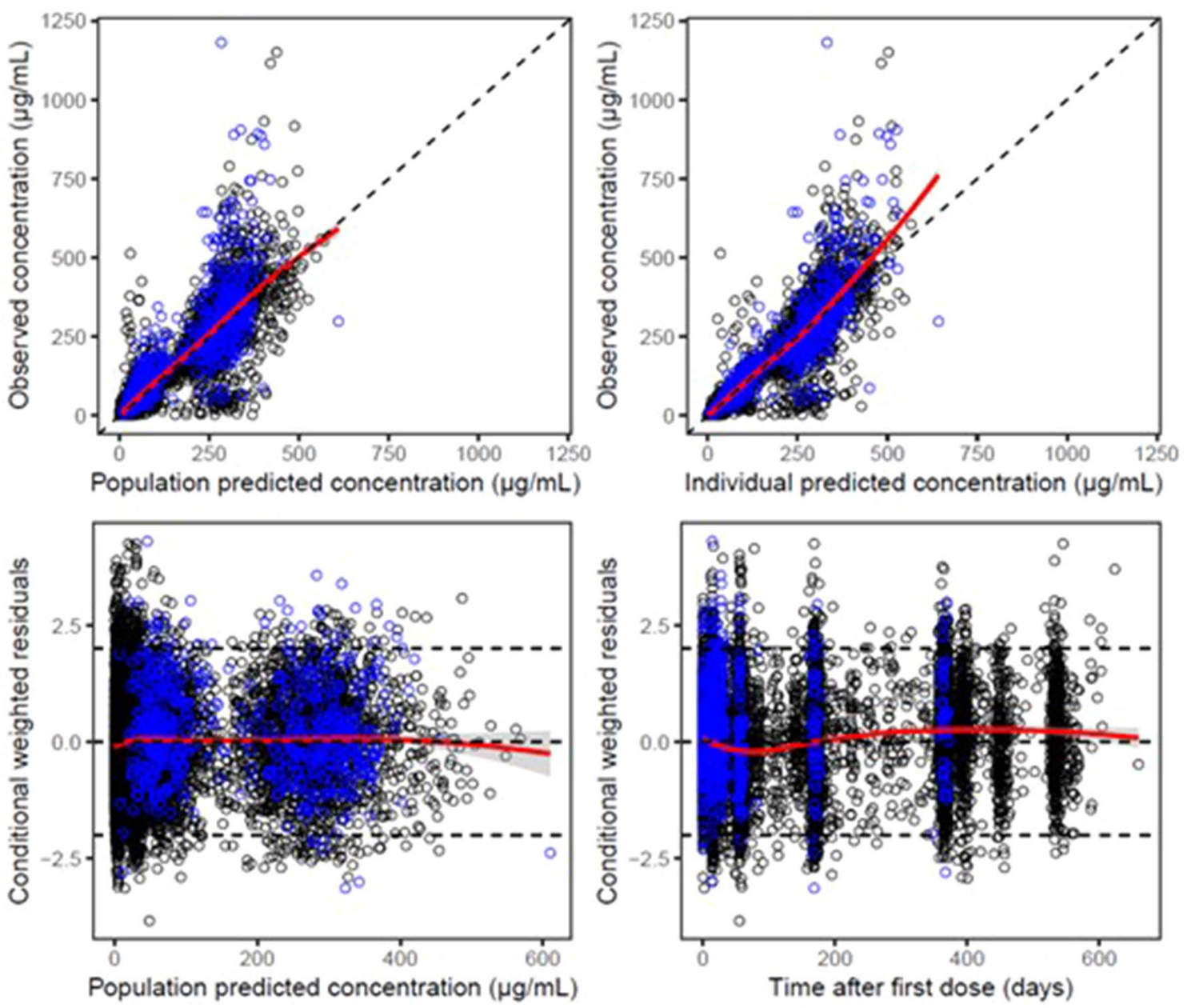

Fig. 3 Goodness-of-fit plots for the final population pharmacokinetic model. Run613. Blue points are pediatric data; black points are adult data; black dashed lines are the identity or zero line; red lines are Loess fit

populations did significantly lower the OFV, the model fit was not obviously improved and the model still overpredicted the data from study BEL113750. One possible reason is a difference in the bioanalysis methods, which were performed in two different laboratories (one for Chinese sites and one for non-Chinese sites). In the cross-validation exercise, the results obtained from the laboratory in China were approximately $7.5 \%$ lower than those from the other laboratory. Ultimately, racial differences within the North East Asian population were not considered in our final model.

The results of this study indicate a degree of uncertainty surrounding the expected exposures in a Chinese pediatric population. A population model fitted to all available adult and pediatric data could not completely explain the different exposures observed between non-Chinese pediatric patients and Chinese adults in study BEL113750. Nevertheless, the model fit to the wider dataset was consistently good across all other studies, including a second pharmacokinetic study in Chinese adults (study 200909). The model is considered to be an accurate and reliable representation of the pharmacokinetics in both adults and pediatrics as well as in the North East Asian and Chinese subgroups. The model can therefore be used with confidence to predict pharmacokinetics in a Chinese pediatric population. 

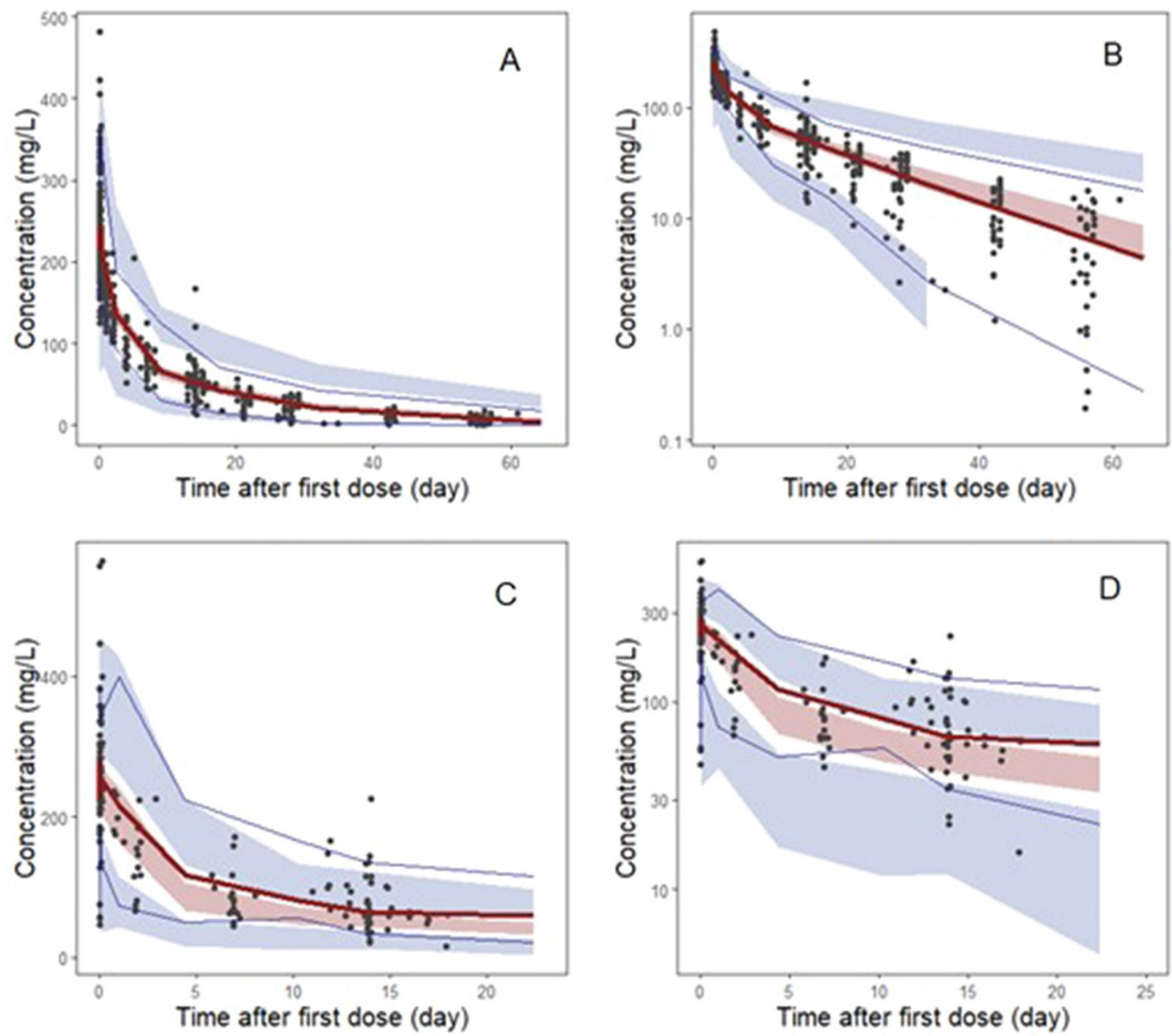

Fig. 4 Visual predictive check of observed vs. simulated data stratified by adult (upper) and pediatric (lower) patients. Left: linear scale, right: $\log$ scale. Black dots are observations; lines are the 5\%,50\%,

and $95 \%$ quantiles of the observations; bands are $95 \%$ confidence intervals of the corresponding quantiles of the simulated model

suggests no great difference in exposure between pediatric and Chinese adult populations. The proposed belimumab $10 \mathrm{mg} / \mathrm{kg}$ IV Q4W dosing regimen for Chinese pediatric patients with SLE should result in similar exposure to that in Chinese adult and non-Chinese pediatric patients, supporting the use of belimumab $10 \mathrm{mg} / \mathrm{kg}$ IV in Chinese pediatric patients with SLE. 

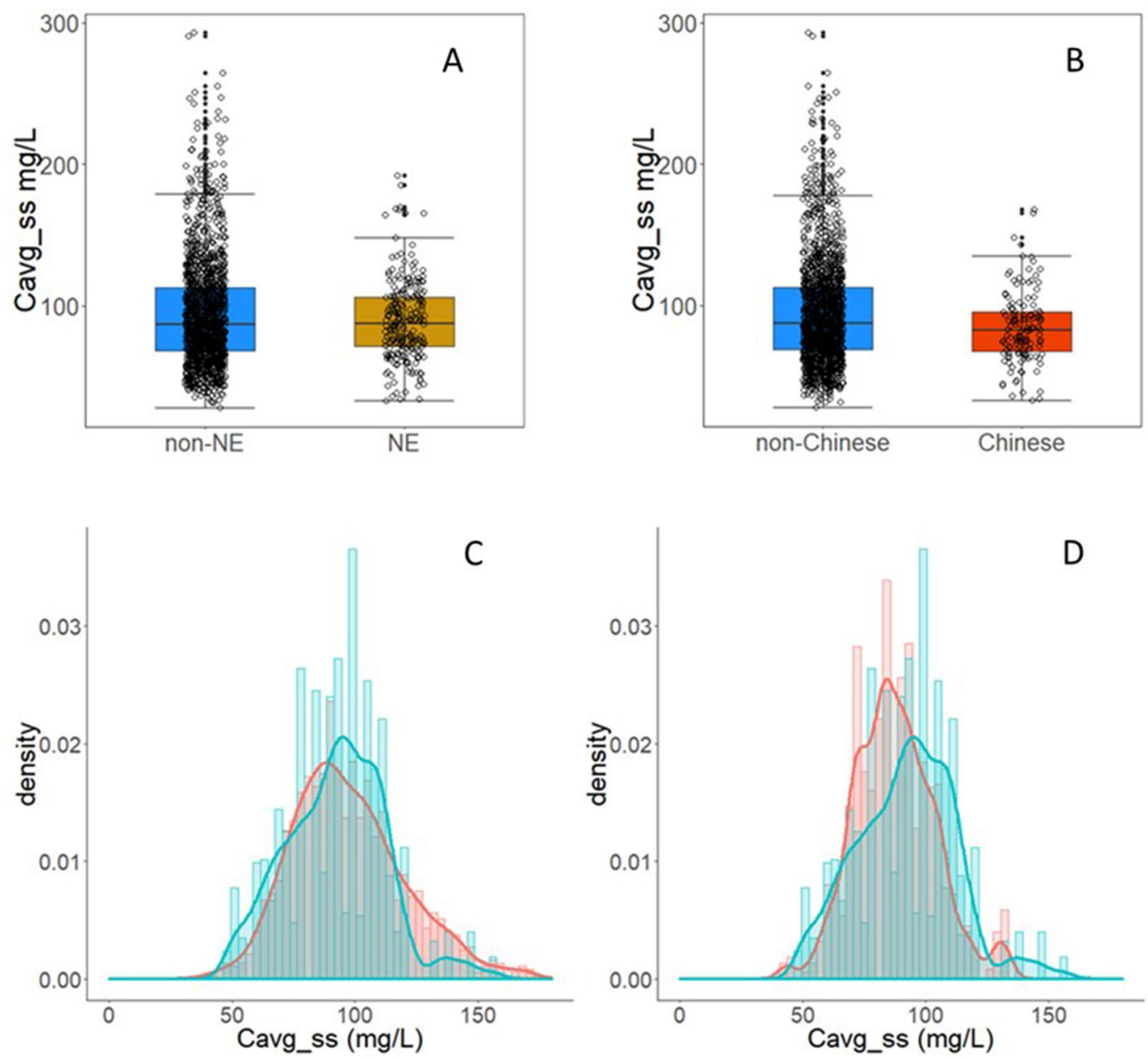

Fig. 5 Pharmacokinetic exposure comparison of (A) North East Asian vs. non-North East Asian populations, (B) Chinese vs. nonChinese populations, (C) simulated pediatric patients (blue) vs. all

Table 3 Distribution of steady-state average concentration $(\mathrm{mg} / \mathrm{l})$ for adult and pediatric patients with systemic lupus erythematosus

\begin{tabular}{lll}
\hline Population & First quartile & Third quartile \\
\hline $\begin{array}{l}\text { All adult patients (80-125\%) } \\
\text { North East Asian adult } \\
\begin{array}{l}\text { patients (80-125\%) } \\
\text { All pediatric patients }\end{array}\end{array}$ & $75.8(64.8-101.3)$ & $111.2(89.0-139.0)$ \\
\hline
\end{tabular}

Supplementary Information The online version contains supplementary material available at https://doi.org/10.1007/s40268-021-00363-2.

Acknowledgements The authors thank Richard Dimelow and Herbert Struemper (GSK) for performing previous modeling analyses based on pediatric and adult data, Xueying Sun and Jialin Xu (GSK) for checking the data set, and Chao Chen (GSK) and Richard Dimelow for reviewing the manuscript.

adults (red), and (D) simulated pediatric patients (blue) vs. simulated North East Asian adults (red). $C_{\text {avg_ss }}$ steady-state average concentration, NE North East Asian populations

\section{Declarations}

Funding This study was funded by GSK, which contributed to the design, collection, analysis, and interpretation of the data; contributed to the decision to submit the paper for publication; and supported the authors in the development of the manuscript. Medical writing support was provided by Olga Conn, PhD, of Fishawack Indicia Ltd., UK, funded by GSK.

Conflicts of interest $\mathrm{XZ}$ is an employee of GSK and holds stocks and shares in the company. T-IL and PM were employees of GSK and held stocks and shares in the company at the time of the study. MZ is an intern of GSK.

Ethics approval Not required for the current study; all anonymized data were aggregated from the original studies.

Consent to participate Not required for the current study; obtained for the original studies. 
Consent for publication All authors approved the content of the submitted manuscript for publication.

Availability of data and material GSK is committed to publicly disclosing the results of GSK-sponsored clinical research that evaluates GSK medicines and as such was involved in the decision to submit. Anonymized individual patient data and study documents can be requested for further research from www.clinicalstudydatarequest.com.

Code availability Available in the electronic supplementary material.

Author contributions $\mathrm{XZ}$ and PM contributed to the conception and design of the study. T-IL and MZ contributed to the data sorting and NONMEM dataset. MZ contributed to the model updating. All authors contributed to data analysis or interpretation.

Open Access This article is licensed under a Creative Commons Attribution-NonCommercial 4.0 International License, which permits any non-commercial use, sharing, adaptation, distribution and reproduction in any medium or format, as long as you give appropriate credit to the original author(s) and the source, provide a link to the Creative Commons licence, and indicate if changes were made. The images or other third party material in this article are included in the article's Creative Commons licence, unless indicated otherwise in a credit line to the material. If material is not included in the article's Creative Commons licence and your intended use is not permitted by statutory regulation or exceeds the permitted use, you will need to obtain permission directly from the copyright holder. To view a copy of this licence, visit http://creativecommons.org/licenses/by-nc/4.0/.

\section{References}

1. Hong SD, Reiff A, Yang HT, Migone TS, Ward CD, Marzan K, Shaham B, Phei WC, Garza J, Bernstein B, et al. B lymphocyte stimulator expression in pediatric systemic lupus erythematosus and juvenile idiopathic arthritis patients. Arthritis Rheum. 2009;60(11):3400-9.

2. Petri M, Stohl W, Chatham W, McCune WJ, Chevrier M, Ryel J, Recta V, Zhong J, Freimuth W. Association of plasma B lymphocyte stimulator levels and disease activity in systemic lupus erythematosus. Arthritis Rheum. 2008;58(8):2453-9.

3. Shin W, Lee HT, Lim H, Lee SH, Son JY, Lee JU, Yoo KY, Ryu SE, Rhie J, Lee JY, et al. BAFF-neutralizing interaction of belimumab related to its therapeutic efficacy for treating systemic lupus erythematosus. Nat Commun. 2018;9(1):1200.

4. Furie R, Petri M, Zamani O, Cervera R, Wallace DJ, Tegzova D, Sanchez-Guerrero J, Schwarting A, Merrill JT, Chatham WW, et al. A phase III, randomized, placebo-controlled study of belimumab, a monoclonal antibody that inhibits B lymphocyte stimulator, in patients with systemic lupus erythematosus. Arthritis Rheum. 2011;63(12):3918-30.

5. Navarra SV, Guzman RM, Gallacher AE, Hall S, Levy RA, Jimenez RE, Li EK, Thomas M, Kim HY, Leon MG, et al. Efficacy and safety of belimumab in patients with active systemic lupus erythematosus: a randomised, placebo-controlled, phase 3 trial. Lancet. 2011;377(9767):721-31.

6. Stohl W, Schwarting A, Okada M, Scheinberg M, Doria A, Hammer AE, Kleoudis C, Groark J, Bass D, Fox NL, et al. Efficacy and safety of subcutaneous Belimumab in Systemic Lupus Erythematosus: a fifty-two-week randomized, double-blind, placebocontrolled study. Arthritis Rheumatol. 2017;69(5):1016-27.

7. Zhang F, Bae SC, Bass D, Chu M, Egginton S, Gordon D, Roth DA, Zheng J, Tanaka Y. A pivotal phase III, randomised, placebo-controlled study of belimumab in patients with systemic lupus erythematosus located in China, Japan and South Korea. Ann Rheum Dis. 2018;77(3):355-63.

8. PI B. Prescribing Information 2019. Available at: https://www. accessdata.fda.gov/drugsatfda_docs/label/2019/125370s064 ,761043s007lbl.pdf. Accessed May 2021.

9. SMPC B. Benlysta Summary of Product Characteristics. Available at: https://www.ema.europa.eu/en/documents/product-infor mation/benlysta-epar-product-information_en.pdf. Accessed May 2021.

10. GSK.: Benlysta, the world's first biologic therapy for the treatment of systemic lupus erythematosus (SLE), now approved in mainland China. https://www.gsk-china.com/en-gb/media/press-relea ses/2019/benlysta-the-world-s-first-biologic-therapy-for-the-treat ment-of-systemic-lupus-erythematosus-sle-now-approved-inmainland-china/. Accessed Nov 2020.

11. PMDA.: Benlysta Report on Deliberation Results Weblink: https:// www.pmda.go.jp/files/000233737.pdf. Accessed Nov 2020.

12. Ruperto N, Abud-Mendoza C, Viola DO, Calvo I, Levy DM, Calderon GJ, Ferrandiz M, Chasnyk V, Keltsev V, Anton J, et al. The PLUTO study: intravenous belimumab in children with systemic lupus erythematosus. Ann Rheum Dis. 2019;78(Suppl 2):764-5.

13. Brunner HI, Abud-Mendoza C, Viola DO, Calvo Penades I, Levy D, Anton J, Calderon JE, Chasnyk VG, Ferrandiz MA, Keltsev $\mathrm{V}$, et al. Safety and efficacy of intravenous belimumab in children with systemic lupus erythematosus: results from a randomised, placebo-controlled trial. Ann Rheum Dis. 2020;79(10):1340-8.

14. Comission E. European Commission approves Benlysta for adult patients with active lupus nephritis [Press release]. 5 May. 2021. Available at: https://www.gsk.com/en-gb/media/press-releases/ european-commission-approvesbenlysta-for-adult-patients-withactive-lupus-nephritis/. Accessed May 2015.

15. Zhang J, Wan W, Miao L, Wu J, Dong J, Shen Y, Xiong C, Li C, Xue Y, Cao G, et al. Pharmacokinetics, pharmacodynamics and safety of Belimumab in Chinese patients with Systemic Lupus Erythematosus: a phase I, open-label study. Rheumatol Ther. 2020;7(1):191-200.

16. Germovsek E, Barker CIS, Sharland M, Standing JF. Pharmacokinetic-pharmacodynamic modeling in pediatric drug development, and the importance of standardized scaling of clearance. Clin Pharmacokinet. 2019;58(1):39-52.

17. Struemper H, Chen C, Cai W. Population pharmacokinetics of belimumab following intravenous administration in patients with systemic lupus erythematosus. J Clin Pharmacol. 2013;53(7):711-20.

18. Struemper H, Thapar M, Roth D. Population pharmacokinetic and pharmacodynamic analysis of Belimumab administered subcutaneously in healthy volunteers and patients with Systemic Lupus Erythematosus. Clin Pharmacokinet. 2018;57(6):717-28.

19. Chaudhury C, Mehnaz S, Robinson JM, Hayton WL, Pearl DK, Roopenian DC, Anderson CL. The major histocompatibility complex-related $\mathrm{Fc}$ receptor for $\mathrm{IgG}(\mathrm{FcRn})$ binds albumin and prolongs its lifespan. J Exp Med. 2003;197(3):315-22.

20. Janmahasatian S, Duffull SB, Ash S, Ward LC, Byrne NM, Green B. Quantification of lean bodyweight. Clin Pharmacokinet. 2005;44(10):1051-65.

21. Haidar SH, Davit B, Chen ML, Conner D, Lee L, Li QH, Lionberger R, Makhlouf F, Patel D, Schuirmann DJ, et al. Bioequivalence approaches for highly variable drugs and drug products. Pharm Res. 2008;25(1):237-41.

22. Yapa SW, Roth D, Gordon D, Struemper H. Comparison of intravenous and subcutaneous exposure supporting dose selection of subcutaneous belimumab systemic lupus erythematosus Phase 3 program. Lupus. 2016;25(13):1448-55.

23. Portet S. A primer on model selection using the Akaike Information Criterion. Infect Dis Model. 2020;5:111-28. 